Published in final edited form as:

Curr Opin Neurol. 2009 August ; 22(4): 340-347. doi:10.1097/WCO.0b013e32832d93dd.

\title{
Human Brain Networks in Health and Disease
}

\author{
Danielle S. Bassett ${ }^{1,2,3}$ and Edward T. Bullmore 2,4 \\ ${ }^{1}$ Behavioral \& Clinical Neurosciences Institute, University of Cambridge, Department of Psychiatry, \\ Addenbrooke's Hospital, Cambridge UK \\ 2Biological Soft Systems Sector, Department of Physics, University of Cambridge, Cambridge UK \\ ${ }^{3}$ Genes Cognition \& Psychosis Program, Clinical Brain Disorders Branch, National Institute of \\ Mental Health, NIH, Bethesda, MD, USA \\ ${ }^{4}$ Clinical Unit Cambridge, GlaxoSmithKline, Addenbrooke's Hospital, Cambridge UK
}

\section{Abstract}

\begin{abstract}
Purpose of review-Recent developments in the statistical physics of complex networks have been translated to neuroimaging data in an effort to enhance our understanding of human brain structural and functional networks. This review focuses on studies using graph theoretical measures applied to structural MRI, diffusion MRI, functional MRI, electroencephalography and magnetoencephalography data.
\end{abstract}

\begin{abstract}
Recent findings-Complex network properties have been identified with some consistency in all modalities of neuroimaging data and over a range of spatial and time scales. Conserved properties include small-worldness, high efficiency of information transfer for low wiring cost, modularity, and the existence of network hubs. Structural and functional network metrics have been found to be heritable and to change with normal aging. Clinical studies, principally in Alzheimer's disease and schizophrenia, have identified abnormalities of network configuration in patients. Future work will likely involve efforts to synthesize structural and functional networks in integrated models and to explore the inter-dependence of network configuration and cognitive performance.
\end{abstract}

Summary-Graph theoretical analysis of neuroimaging data is growing rapidly and could potentially provide a relatively simple but powerful quantitative framework to describe and compare whole human brain structural and functional networks under diverse experimental and clinical conditions.

\section{Keywords}

network; graph; small-world; modularity; wiring cost

\section{Introduction}

The concept of networks in neurology originated in the latter half of the $19^{\text {th }}$ century with the advent of the "disconnection syndromes" hypothesis propounded by Wernicke, Lichtheim, Liepmann, Dejerine and others mainly on the basis of clinico-pathological correlations. The concept was fostered by two developments in related fields: 1) the movement from a

Correspondence and requests for materials should be addressed to E.T.B. (etb23@cam.ac.uk).

Publisher's Disclaimer: This is a PDF file of an unedited manuscript that has been accepted for publication. As a service to our customers we are providing this early version of the manuscript. The manuscript will undergo copyediting, typesetting, and review of the resulting proof before it is published in its final citable form. Please note that during the production process errors may be discovered which could affect the content, and all legal disclaimers that apply to the journal pertain. 
characterization of human brain surface morphology towards a direct description of neuroanatomical projection pathways in the white matter and 2) the widespread acceptance of "associationistic" models of cognitive function described by James and Freud [1]. However, the early "diagram-makers", as they were called by their detractors, lost ground to competing theories of brain organization (such as mass action) in the mid-20th century. The central concept of a brain network constructed to segregate and integrate information processing was not again influential until reintroduced to the English speaking world by Norman Geschwind in the 1960s [2,3]. While Geschwind's legacy encouraged deeper exploration into corticocortical connectivity organization, noninvasive imaging techniques to probe these relationships in vivo were not yet widely available.

In the past few decades, a plethora of multiscale noninvasive structural and functional neuroimaging techniques have become cost-effective while the basic data preprocessing methods for these techniques have become more finely tuned. Simultaneously, developments within the realm of statistical physics have led to the formation of a new interdisciplinary field, now known as "complex network science", which provides mathematical and conceptual tools for understanding the organization and emergent behaviors of a diverse range of complex reallife networks; see [4] for an accessible introduction and [5] for a comprehensive review of the technical background. A basic insight to have emerged already from complex network science is that substantively different systems can share key organizational principles in common.

Moreover, within a complex system like the brain, which exists on many different scales of space and time, it seems that network properties can be highly conserved or scale-invariant. For example, both microscopic cellular networks and macroscopic networks derived from neuroimaging data can demonstrate isomorphic properties such as modularity, the existence of hub nodes, and high efficiency of information transfer for nearly minimal wiring costs; see $[6,7]$ for recent reviews of complex networks in relation to neuroscience generally. In this article we will focus specifically on recent studies using mathematical tools drawn mainly from graph theory to elucidate the complex network properties of the human brain in health and disease.

\section{Graph theoretical concepts for network analysis}

In graph theory, a network is reduced to an abstract description as a set of nodes connected by edges (or lines); see Figure 1. The edges can be directed or undirected, and weighted or unweighted. Most graph theoretical analysis of brain networks to date has considered the simplest case of an undirected, unweighted graph.

The nodes and edges of a brain graph can be empirically defined in many ways. For networks constructed from microscopic data, such as studies on the nematode worm Caenorhabditis elegans [8], the nodes would naturally be neurons and the edges would represent the axons connecting neurons to each other. On the other hand, to construct human brain networks at the macroscopic scale of neuroimaging data, we might specify that the nodes were the major subcortical nuclei and cortical regions and the edges represented some statistical measure of association, e.g., correlation or mutual information, between regions $[9,10]$; see Figure 2 .

Once the network has been rendered in graphical form, its topological properties can be measured. A key topological metric is the degree of each node, which is simply the number of edges connecting it to the rest of the network. The probability distribution of degree over all nodes in the network is called the degree distribution and often has a more-or-less truncated power law form compatible with the existence of high degree nodes or network hubs.

Another key metric is the path length which is the number of edges that must be traversed to go from one node to any other node; for a pair of nodes that are nearest neighbors the path length is 1 ; the average path length over all possible pairs of nodes, sometimes called the 
characteristic path length, is inversely related to the global efficiency of the network for parallel information transfer [11]. The clustering coefficient is a measure of the density of connections between nearest neighbors of an index node: high clustering coefficients indicate nodes that are part of a clique of densely inter-connected neighbors. These and other metrics on brain networks can be compared to their values in benchmark networks, such as random networks (which have low clustering and short path length) or regular lattices (which have high clustering and long path length).

Watts and Strogatz, in their highly influential article in 1998 [12], used a simple computational model to show that between the limiting cases of a regular lattice and a random network there exists an intermediate regime of networks characterized by the combination of high clustering and short path length. They called this class of networks "small world", based on previous observations from sociology that social networks often have similar properties of high clustering or cliquishness of relationships in local neighborhoods, combined with a surprisingly short chain of intermediate acquaintances between any two people selected at random from a large population. This analysis has proven to be attractive to systems neuroscientists because it resolves a long-standing tension between localized and distributed models of brain organization: in principle, a small-world network can provide a topological substrate for both locally specialized or segregated processing in neighborhoods of highly clustered nodes, and globally distributed or integrated processing on a highly efficient network with short characteristic path length [13]. Recent work has considerably extended the range of metrics that have been drawn from complex networks science to applications in neuroscience and neuroimaging; see Table 1.

\section{Structural brain networks}

Human brain structural networks have been constructed in two ways: either indirectly from inter-regional covariation of gray matter volume or thickness measurements in structural magnetic resonance imaging data, sMRI; or more directly from measurements of white matter connections between gray matter regions provided by diffusion tensor imaging, DTI (or related techniques). Nodes of structural networks have usually been defined as regions of a predetermined anatomical parcellation scheme, such as the automated anatomical labeling (AAL) template image, which divides the cortex into approximate Brodmann areas [18]. To make gray matter networks, based on sMRI data, the edges between nodes are defined by the strength of correlation between regional volume or cortical thickness measurements [42]. For example, in a sample of say 100 people, if there is a strong correlation between cortical thickness in right and left middle frontal gyrus, an edge would be drawn between these nodes in an undirected graph representing the sample mean network. The main advantage of white matter networks, based on DTI, is that techniques such as probabilistic tractography can be used to assign a connection probability between any pair of regional nodes in a single subject.

Moreover, anatomical networks based on tractography in a single subject may seem more straightforwardly related to axonal projections between regions than networks based on interregional covariation of gray matter (although see Lerch et al [43] for cross-validation of these two approaches). However, one currently challenging issue for DTI-based networks is that most tractography techniques under-estimate the probability of connections between regions widely separated in space, leading to a relative sparsity of long distance edges in the resulting networks.

\section{Gray matter networks}

Gray matter networks in healthy volunteers have been shown to have small-world topology and relatively low wiring costs, i.e., the mean physical distance between connected nodes is considerably less than in a comparable random network, consistent with prior evidence that 
nervous systems are organized to nearly minimize wiring costs $[14,15]$. In normal multimodal cortical networks, a hierarchical organization was demonstrated, with the highest degree nodes having low clustering; transmodal and unimodal cortical networks were less hierarchically organized, suggesting that different cortical divisions might have developed according to different growth rules [14]. There have also been studies of the modular organization of gray matter networks, showing that these can be decomposed into a community of sparsely interconnected modules, each of which comprises a number of densely intra-connected brain regions [16]. The modular organization of human brain structural networks seems to reflect known functional specializations of the brain regions and is comparable to the modularity of functional networks derived from fMRI [10,21]. A twin design has been used to determine a network of regional structural associations which are under robust genetic control [17], as also validated in functional networks [37].

A study of people with schizophrenia found that their gray matter network was characterized by an increased physical distance between connected nodes, suggestive of inefficient wiring, and attenuated hierarchical organization of heteromodal cortex, which might be indicative of abnormal neurodevelopment [14]. In Alzheimer's disease, gray matter networks have been associated with changes in the degree distribution associated with a more lattice-like network which is highly sensitive to computationally simulated lesions to the hub nodes $[15,44]$.

\section{White matter networks}

Three diffusion-based methods have been used in important studies recently to construct white matter networks: diffusion tensor imaging (DTI) [18], diffusion spectrum imaging (DSI) [19], and diffusion weighted magnetic resonance imaging (DW-MRI) [20]. Convergently, these studies suggest that there exists a core of the white matter network which densely interconnects the posterior and medial cortical regions [19], association cortical hubs [20], and has longer-range white matter connections to the rest of the brain [18].

\section{Functional brain networks}

Functional brain networks have been constructed from functional magnetic resonance imaging (fMRI), electroencephalography (EEG), and magnetoencephalography (MEG).

\section{fMRI}

Functional MR imaging has coarse time resolution (order of seconds) but good spatial resolution (millimeters). It has therefore been used to make anatomically precise, even voxel level $[26,28]$, maps of functional networks operating at low frequencies $(<0.5 \mathrm{~Hz})$.

Two studies this year have confirmed the modular [21] and, in fact, hierarchically modular [22] community structure of healthy functional networks. Meunier et al.(2009) further studied the effect of aging on modular structure, finding an increased number of smaller modules and fewer inter-modular connections to frontal regions in the older adults (mean age $=67.3$ years) than in the younger adults (mean age $=24.3$ years) [21].

Several recent studies have described the effects of diseases such as schizophrenia, attention deficit hyperactivity disorder (ADHD), and Alzheimer's disease (AD) on fMRI brain networks. Liu et al. (2008) showed that topological measurements such as clustering and small-worldness were inversely correlated with duration of illness in schizophrenia [23]. Supekar et al. (2008) showed that the clustering coefficient was significantly reduced in patients with Alzheimer's disease, and could be used to distinguished AD participants from the controls with a sensitivity of $72 \%$ and specificity of $78 \%$ [24]. Interestingly, Buckner et al. further showed that the cortical hubs (nodes with high degree) of the resting state functional network also show high amyloid$\beta$ deposition in people with Alzheimer's disease [28]; see Figure 3. Children with ADHD seem 
to show the opposite trend, with a significantly increased local efficiency (or clustering) compared to age-matched volunteers, with regional changes in efficiency found especially in prefrontal, temporal, and occipital cortices [25].

\section{EEG and MEG}

EEG and MEG data contain information about the brain's electromagnetic activity over a wide range of frequencies $(\sim 1$ to $100 \mathrm{~Hz})$ with millisecond time resolution but relatively poor spatial resolution (centimeters).

Several studies in aging, schizophrenia, and Alzheimer's disease have found functional networks operating in these higher frequencies which show comparable topological changes to those described in the lower frequency fMRI data. For example, the transition from childhood ( $8-12 \mathrm{yr}$ ) to adulthood (21-26 yr) is suggested by Micheloyannis et al. to be characterized by a reduction of overall connectivity (decreased clustering and increased path length) [29]. Similarly, Stam et al. showed, in an analysis of weighted networks derived from resting state MEG data, that patients with Alzheimer's disease had reduced connectivity as shown by decreased clustering and increased path length [40]. Finally, a comparable decrease in local clustering was found in weighted networks derived from EEG data on patients with schizophrenia studied both under resting conditions [30] and while performing a working memory task [31].

The effects of sleep on functional networks have been described in studies using EEG with a relatively small number of electrodes (12-19), resulting in networks that are roughly an order of magnitude smaller than those considered in fMRI and waking electrophysiological techniques. Ferri et al. (2008) reported that small-worldness became steadily greater through light sleep, slow-wave sleep, and REM sleep in frequency bands less than $15 \mathrm{~Hz}$, indicating a definite reconfiguration which may be related to neural plasticity during sleep [32]. Dimitriadis et al. make the further claim that the topological structure of networks in stages 1,2,3,4, and REM are significantly different and can be clearly distinguished with a high sensitivity and specificity using a data driven clustering algorithm [33]. In addition to studies of healthy subjects, Leistedt et al. analyzed the sleep networks of acutely depressed patients and showed that they were characterized by an increased path length, i.e., a randomization of network topology, which may directly impact the cognitive capacity of the brain during wakefulness [34].

\section{Recent challenges and trends}

Given the recent explosion of human brain network papers based on graph theoretical analysis of neuroimaging data, a current challenge to the field is to evaluate the convergence of structural and functional networks measured at different frequency and spatial scales using different techniques. Preliminary evidence suggests anecdotally that there is some degree of isomorphism between gray matter and white matter networks, and between disease-related changes in low and high frequency functional networks derived from fMRI and EEG/MEG data, respectively. However, these issues need to be addressed more rigorously and directly, for example by using computational models [45], to provide a more integrated account of brain network organization [46].

Another emerging issue is that we do not yet understand the impact of different methodological choices at several steps of network generation and analysis on the resultant findings. Studies have used different parcellation schemes [26,27], continuous metrics of association [47], edge weights (continuous or binary) [23,30,38,40], and strategies for thresholding association matrices. Encouragingly, many headline results (e.g., network small-worldness) seem to be 
robust to methodological details but, nonetheless, it will be important to develop a more rational basis for choosing between alternative options.

Within the past year, we have also seen an increasing interest in studying how networks change, e.g., over time and in response to task demands. While network dynamics seem to have a persistent or long memory component [39], they can also adjust quickly to behavioral changes or cognitive demands [35]. This adaptivity to changing environmental contingencies may be related to evidence that brain networks are dynamically in a critical state, "on the edge of chaos", which facilitates their rapid reconfiguration in response to altered inputs [48]. The inter-dependence of network organization and behavior has already been studied for several specific tasks [29,31,36,49,50]. Recent papers have described methodological developments which could be relevant to more extensive applications of graph theoretical analysis to taskrelated functional networks in future [41,51,52].

\section{Conclusion}

Graph theoretical analysis of human brain network organization based on neuroimaging data has developed rapidly in the last 1-2 years. The basic concepts and techniques have proven to be generally applicable to all major current modalities of neuroimaging data over a wide range of spatial and frequency scales. Preliminary data have also indicated that structural and functional network measures are heritable, abnormal in clinical disorders, and change in the context of normal aging, collectively suggesting that these metrics are capturing aspects of brain organization that are of substantive neurobiological importance.

\section{Acknowledgments}

This research was supported by a Human Brain Project grant from the National Institute of Biomedical Imaging \& Bioengineering and the National Institute of Mental Health, and by the Intramural Research Program of the National Institutes of Health, NIMH. D.S.B. was supported by the National Institutes of Health Graduate Partnerships Program. Correspondence and requests for materials should be addressed to E.T.B. (etb23@cam.ac.uk).

\section{References and recommended reading}

Papers of particular interest, published within the annual period of review, have been highlighted as:

* of special interest

** of outstanding interest

1. Catani M, Mesulam M. What is a disconnection syndrome? Cortex. 2008 In Press. * This comment gives a brief history of the concept of a disconnection syndrome.

2. Geschwind N. Disconnexion syndromes in animals and man. I. I. Brain 1965;88:237-294. [PubMed: 5318481]

3. Geschwind N. Disconnexion syndromes in animals and man. II. Brain 1965;88:585-644. [PubMed: 5318824]

4. Barabási AL, Bonabeau E. Scale-free networks. Sci Am 2003;288(5):60-69.

5. Albert R, Barabási AL. Statistical mechanics of complex networks. Rev Mod Phys 2002;74:47-98.

6. Bassett DS, Bullmore ET. Small-world brain networks. Neuroscientist 2006;12:512-523. [PubMed: 17079517]

7. Bullmore E, Sporns O. Complex brain networks: graph theoretical analysis of anatomical and functional systems. Nature Reviews Neuroscience. 2009 In Press. ** This article is both a comprehensive review of the history of the field of complex brain networks and a useful delineation of recent developments. 
8. Chatterjee N, Sinha S. Understanding the mind of a worm: hierarchical network structure underlying nervous system function in C. elegans. Prog Brain Res 2008;168:145-153. [PubMed: 18166392]* This work studies the hierarchical network architecture of C. elegans.

9. Achard S, Salvador R, Whitcher B, et al. A resilient, low-frequency, small-world human brain functional network with highly connected association cortical hubs. J Neurosci 2006;26(1):63-72. [PubMed: 16399673]

10. Salvador R, Suckling J, Schwarzbauer C, Bullmore ET. Undirected graphs of frequency-dependent functional connectivity in whole brain networks. Philos Trans R Soc Lond B Biol Sci 2005;360 (1457):937. [PubMed: 16087438]

11. Latora V, Marchiori M. Efficient behavior of small-world networks. Phys Rev Lett 2001;87 198701.

12. Watts DJ, Strogatz SH. Collective dynamics of 'small-world' networks. Nature 1998;393(6684):440442. [PubMed: 9623998]

13. Sporns O, Zwi J. The small world of the cerebral cortex. Neuroinformatics 2004;2:145-162. [PubMed: 15319512]

14. Bassett DS, Bullmore ET, Verchinski BA, et al. Hierarchical organization of human cortical networks in health and schizophrenia. J Neurosci 2008;28(37):9239-9248. [PubMed: 18784304] ** This is the first exploration of structural whole brain networks derived from covariation in gray matter volumes.

15. He Y, Chen ZJ, Evans AC. Structural insights into aberrant topological patterns of large-scale cortical networks in Alzheimer's disease. J Neurosci 2008;28(18):4756-4766. [PubMed: 18448652] ** This is the first work to study the structural networks of people with Alzheimer's disease as derived from covariation in cortical thickness.

16. Chen ZJ, He Y, Rosa-Neto P, et al. Revealing modular architecture of human brain structural networks by using cortical thickness from MRI. Cereb Cortex 2008;18(10):2374-2381. [PubMed: 18267952] * This work provides the first analysis of the modular architecture of structural human brain networks.

17. Schmitt JE, Lenroot RK, Wallace GL, et al. Identification of genetically mediated cortical networks: a multivariate study of pediatric twins and siblings. Cereb Cortex 2008;18(8):1737-1747. [PubMed: 18234689 ] ** This work is the first to determine that there is a network of structural connections which are under robust genetic control.

18. Gong G, He Y, Concha L, et al. Mapping anatomical connectivity patterns of human cerebral cortex using in vivo diffusion tensor imaging tractography. Cereb Cortex 2009;19(3):524-536. [PubMed: 18567609] * This work provides the largest sample to date of healthy brain structural networks derived from diffusion tensor imaging deterministic tractography ( $\mathrm{N}=80$ young adults).

19. Hagmann P, Cammoun L, Gigandet X, et al. Mapping the structural core of human cerebral cortex. PLoS Biol 2008;6(7):e159. [PubMed: 18597554]* This is the first work to use diffusion spectrum imaging in a small group ( $\mathrm{N}=5)$ of healthy adults to construct structural brain networks. This work describes a topological structural core of the brain's anatomical connections which contains the posterior components of the human functional default mode network.

20. Iturria-Medina Y, Sotero RC, Canales-Rodríguez EJ, et al. Studying the human brain anatomical network via diffusion-weighted MRI and graph theory. Neuroimage 2008;40(3):1064-1076.

[PubMed: 18272400]* This is the first study to use diffusion-weighted MRI to construct structural brain networks from a cohort of healthy adults.

21. Meunier D, Achard S, Morcom A, Bullmore ET. Age-related changes in modular organization of human brain functional networks. Neuroimage 2009;44:715-723. [PubMed: 19027073] * This work describes the top level modular organization of a group of $\mathrm{N}=17$ healthy young subjects and characterizes the effects of aging on this organization using a group of $\mathrm{N}=13$ older subjects.

22. Ferrarini L, Veer IM, Baerends E, et al. Hierarchical functional modularity in the resting-state human brain. Hum Brain Mapp. 2008 (online). * This work describes the hierarchical modular structure of resting fMRI networks constructed using a partial correlation analysis.

23. Liu Y, Liang M, Zhou Y, et al. Disrupted small-world networks in schizophrenia. Brain 2008;131:945-961. [PubMed: 18299296] * This is the first study to show evidence for altered network topology in the resting state connectivity of people with schizophrenia.

24. Supekar K, Menon V, Rubin D, et al. Network analysis of intrinsic functional brain connectivity in Alzheimer's disease. PLoS Comput Biol 2008;4(6):e1000100. [PubMed: 18584043] * This work 
studies 21 people with Alzheimer's and 18 controls and shows that Alzheimer's disease is characterized by a decrease in local efficiency and an increase in global efficiency, suggesting a more regular 'lattice'-like topology.

25. Wang L, Zhu C, He Y, et al. Altered small-world brain functional networks in children with attentiondeficit/hyperactivity disorder. Hum Brain Mapp 2009;30(2):638-649. [PubMed: 18219621] * This work studies brain networks derived from resting fMRI data in children with ADHD and shows that in comparison to controls, they have significantly higher local clustering.

26. van den Heuvel MP, Stam CJ, Boersma M, Hulshoff HE. Small-world and scale-free organization of voxel-based resting-state functional connectivity in the human brain. Neuroimage 2008;43(3):528539. [PubMed: 18786642]

27. Wang J, Wang L, Zang Y, et al. Parcellation-dependent small-world brain functional networks: a resting-state fMRI study. Hum Brain Mapp. 2008 Epub ahead of print. * This work compares resting state fMRI networks in healthy controls derived from two different parcellation schemes.

28. Buckner RL, Sepulcre J, Talukdar T, et al. Cortical hubs revealed by intrinsic functional connectivity: mapping, assessment of stability, and relation to Alzheimer's disease. J Neurosci 2009;29(6):18601873. [PubMed: 19211893] ** This study first describes the stability of cortical hubs in a large group of healthy subjects in both resting and task conditions (fMRI), and then uses PET to show high amyloid- $\beta$ deposition in these hubs in people with Alzheimer's disease.

29. Micheloyannis S, Vourkas M, Tsirka V, et al. The influence of ageing on complex brain networks: a graph theoretical analysis. Hum Brain Mapp 2009;30(1):200-208. [PubMed: 17990300]* This study shows that the transition from childhood to adulthood is characterized by an overall reduced connectivity in resting EEG functional networks.

30. Rubinov M, Knock SA, Stam CJ, et al. Small-world properties of nonlinear brain activity in schizophrenia. Hum Brain Mapp 2009;30(2):403-416. [PubMed: 18072237] * This work studies EEG networks whose connections are weighted by the strength of nonlinear association between the two regions and specifically in relation to schizophrenia.

31. Pachou E, Vourkas M, Simos P, et al. Working memory in schizophrenia: an EEG study using power spectrum and coherence analysis to estimate cortical activation and network behavior. Brain Topogr 2008;21(2):128-137. [PubMed: 18726681] * This work is the first to describe EEG network properties of working memory connectivity in schizophrenia.

32. Ferri R, Rundo F, Bruni O, et al. The functional connectivity of different EEG bands moves towards small-world network organization during sleep. Clin Neurophysiol 2008;119(9):2026-2036.

[PubMed: 18571469] * This work characterizes the small-world topology of sleep EEG networks in light, slowwave, and REM sleep in a variety of frequency bands of less than $30 \mathrm{~Hz}$.

33. Dimitriadis SI, Laskaris NA, Del Rio-Portilla Y, Koudounis GC. Characterizing dynamic functional connectivity across sleep stages from EEG. Brain Topogr. 2008 Epub Ahead of Print. * This work studies the topological independence of EEG networks constructed in Stages 1, 2, 3, 4, and REM sleep for a small sample of 10 healthy controls.

34. Leistedt SJ, Coumans N, Dumont M, et al. Altered sleep brain functional connectivity in acutely depressed patients. Hum Brain Mapp. 2008 Epub Ahead of Print. * This is the first work to study EEG sleep networks in acutely depressed patients.

35. De Vico Fallani F, Astolfi L, Cincotti F, et al. Cortical network dynamics during foot movements. Neuroinformatics 2008;6(1):23-34. [PubMed: 18266112] * This work provides the first description of networks that change in time in relation to a behavior, which in this case is a simple foot movement.

36. Astolfi L, De Vico Fallani F, Cincotti F, et al. Neural basis for brain responses to TV commercials: a high-resolution EEG study. IEEE Trans Neural Syst Rehabil Eng 2008;16(6):522-531. [PubMed: 19144584]

37. Smit DJ, Stam CJ, Posthuma D, et al. Heritability of "small-world" networks in the brain: a graph theoretical analysis of resting-state EEG functional connectivity. Hum Brain Mapp 2008;29(12): 1368-1378. [PubMed: 18064590] ** This work is the first to show that network architecture in functional EEG resting networks is under genetic control.

38. Ponten SC, Douw L, Bartolomei F, et al. Indications for network regularization during absence seizures: Weighted and unweighted graph theoretical analyses. Exp Neurol. 2009 Epub ahead of print. * This work suggests that brain functional EEG networks become more regularized during absence seizures. 
39. Achard S, Bassett DS, Meyer-Lindenberg A, Bullmore ET. Fractal connectivity of long-memory networks. Phys Rev E 2008;77 036104.

40. Stam CJ, de Haan W, Daffertshofer A, et al. Graph theoretical analysis of magnetoencephalographic functional connectivity in Alzheimer's disease. Brain 2009;132(1):213-224. [PubMed: 18952674]

* This work provides added evidence that Alzheimer's disease functional networks are characterized by a decreased local connectivity structure.

41. Valencia M, Martinerie J, Dupont S, Chavez M. Dynamic small-world behavior in functional brain networks unveiled by an event-related networks approach. Phys Rev E 2008;77(5) 050905.

42. He Y, Chen ZJ, Evans AC. Small-world anatomical networks in the human brain revealed by cortical thickness from MRI. Cereb Cortex 2007;17:2407-2419. [PubMed: 17204824]

43. Lerch JP, Worsley K, Shaw GP, et al. Mapping anatomical correlations across cerebral cortex (MACACC) using cortical thickness from MRI. Neuroimage 2006;31:993-1003. [PubMed: 16624590]

44. Riedl V, Honey CJ. Alzheimer's disease: a search for broken links. J Neurosci 2008;28(33):81488149. [PubMed: 18701676]

45. Honey CJ, Sporns O, Cammoun L, et al. Predicting human resting-state functional connectivity from structural connectivity. Proc Natl Acad Sci U S A 2009;106(6):2035-2040. [PubMed: 19188601] ** This work combines experiment and computational simulations to explore directly the relationship between structural and functional connectivity architectures.

46. Rykhlevskaia E, Gratton G, Fabiani M. Combining structural and functional neuroimaging data for studying brain connectivity: a review. Psychophysiology 2008;45(2):173-187. [PubMed: 17995910]

47. David O, Cosmelli D, Friston KJ. Evaluation of different measures of functional connectivity using a neural mass model. Neuroimage 2004;21:659-673. [PubMed: 14980568]

48. Kitzbichler MG, Smith M, Christensen S, Bullmore ET. Broadband criticality of human brain network synchronization. PLoS Comput Biol. 2009 In press.

49. Bassett DS, Meyer-Lindenberg A, Achard S, et al. Adaptive reconfiguration of fractal small-world human brain functional networks. Proc Natl Acad Sci USA 2006;103:19518-19523. [PubMed: 17159150]

50. Toro R, Fox PT, Paus T. Functional coactivation map of the human brain. Cereb Cortex 2008;18(11): 2553-2559. [PubMed: 18296434]

51. Dosenbach NU, Fair DA, Cohen AL, et al. A dual-networks architecture of top-down control. Trends Cogn Sci 2008;12(3):99-105. [PubMed: 18262825]

52. Fair DA, Schlaggar BL, Cohen AL, et al. A method for using blocked and event-related fMRI data to study "resting state" functional connectivity. Neuroimage 2007;35(1):396-405. [PubMed: 17239622] 

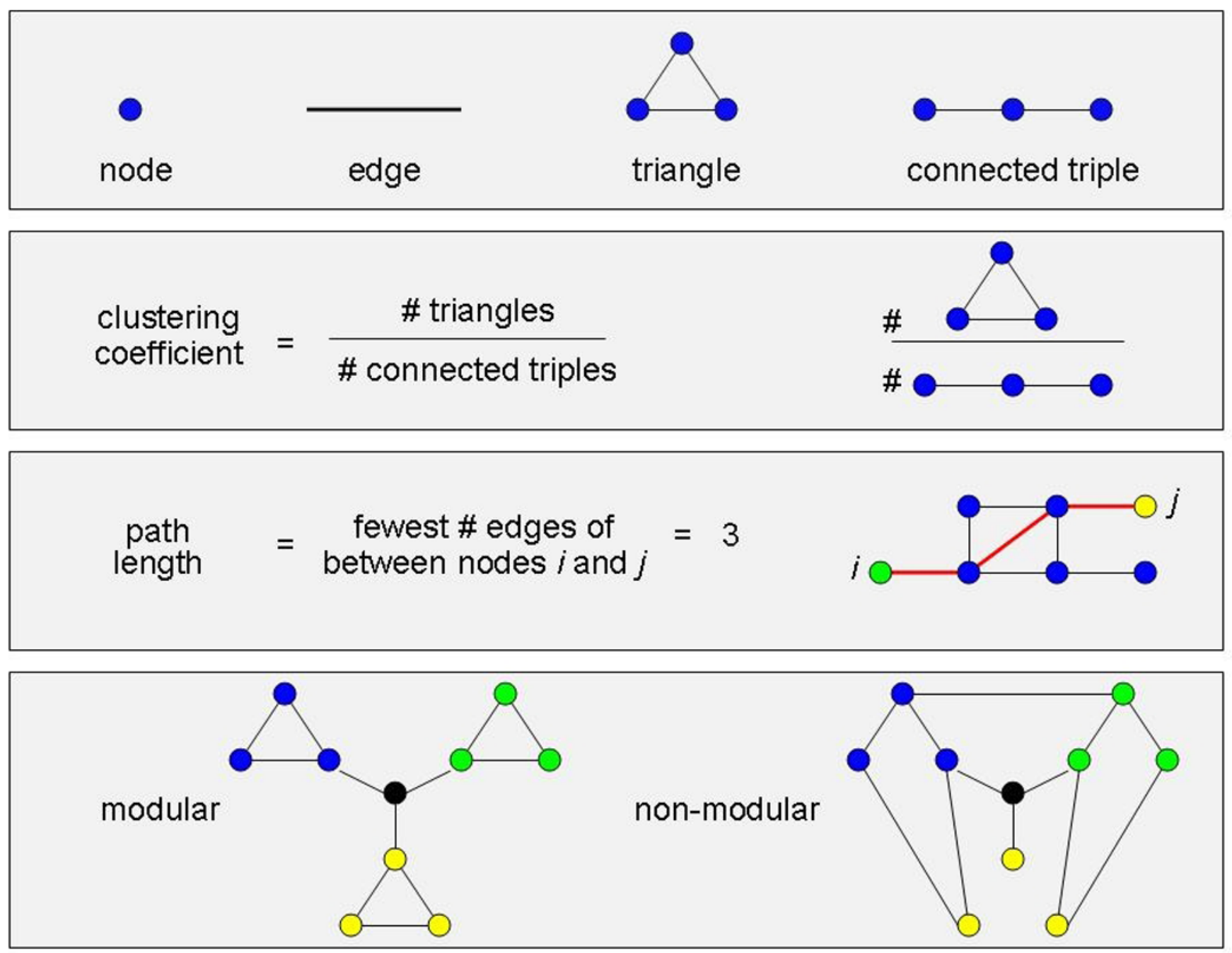

Figure 1.

Tutorial of basic network concepts. Top Plaque Definitions for a node, an edge, a triangle, and a connected triple. Second Plaque The clustering coefficient, $C$, is given by the ratio of the number of connected triangles to the number of connected triples. Third Plaque The path length, $L$, is given by the fewest number of edges linking one node, $i$, to another node, $j$. Bottom Plaque A modular network structure occurs when there are more connections within a module than between modules. In this schematic, modules are given by distinct colors, e.g., blue, green, and yellow. 


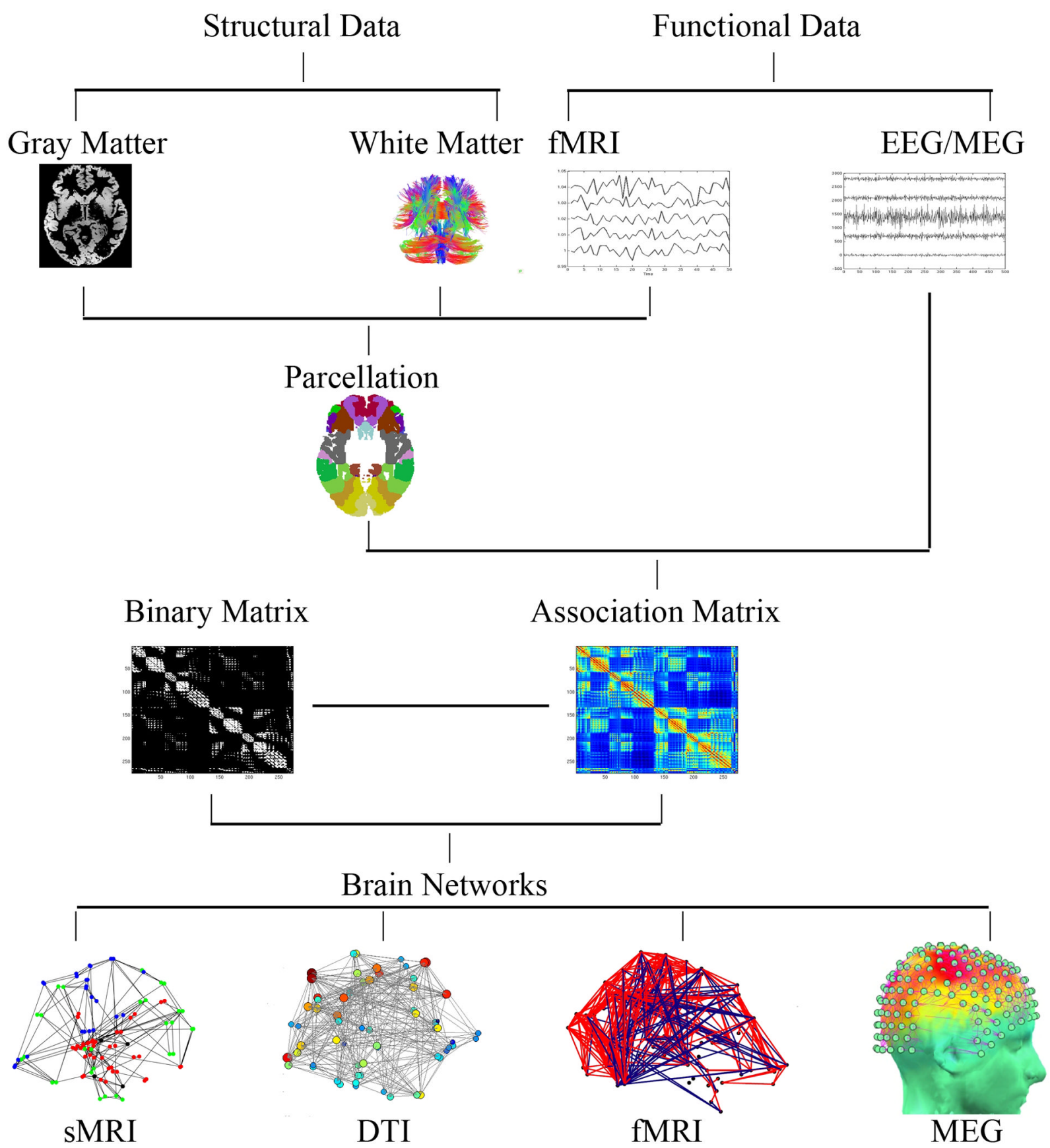

Figure 2.

Workflow of human brain network construction. To construct a brain network, one can begin with either structural (including either gray or white matter measurements) or functional data (including low frequency fMRI data and high frequency EEG or MEG data). Raw data is conventionally put into a parcellation scheme whereby the brain is subdivided into on the order of 100 regions of interest. For EEG and MEG data, this parcellation is already performed by the sensors. The pairwise association between brain regions is then computed, and usually thresholded to create a binary matrix. A brain network is then constructed from nodes (brain regions) and edges (pairwise associations which were larger than the chosen threshold). 
CORTIĆAL HÜBS
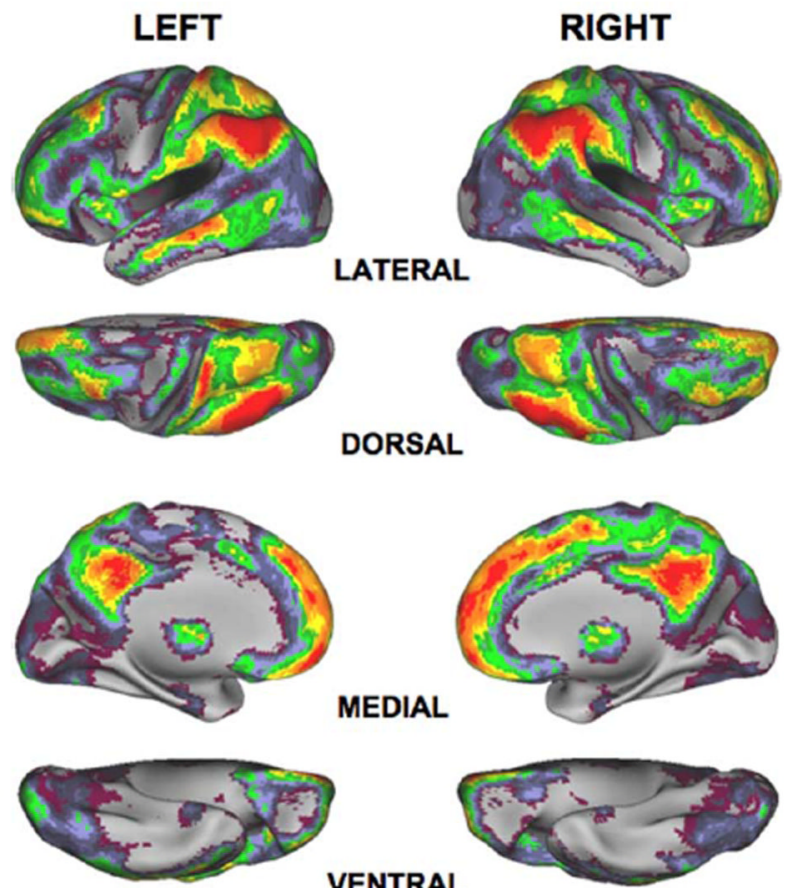

VENTRAL

0.0

\section{A $\beta$ DEPOSITION}
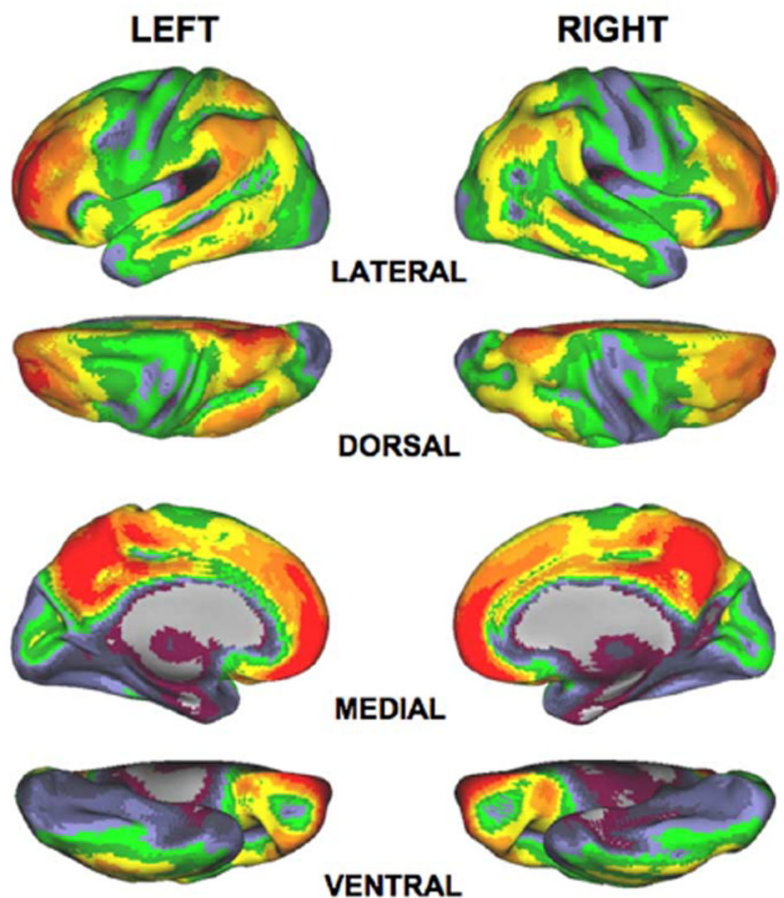

VENTRAL

0.0

Figure 3.

Network hubs have increased amyloid- $\beta$ deposition in Alzheimer's disease. Left Location of cortical hubs, i.e., nodes with a high number of connections or degree, in healthy resting state fMRI networks. Right Location of greatest amyloid- $\beta$ deposition in people with Alzheimer's disease as measured in a PET study. Reproduced with permission from [28]. 


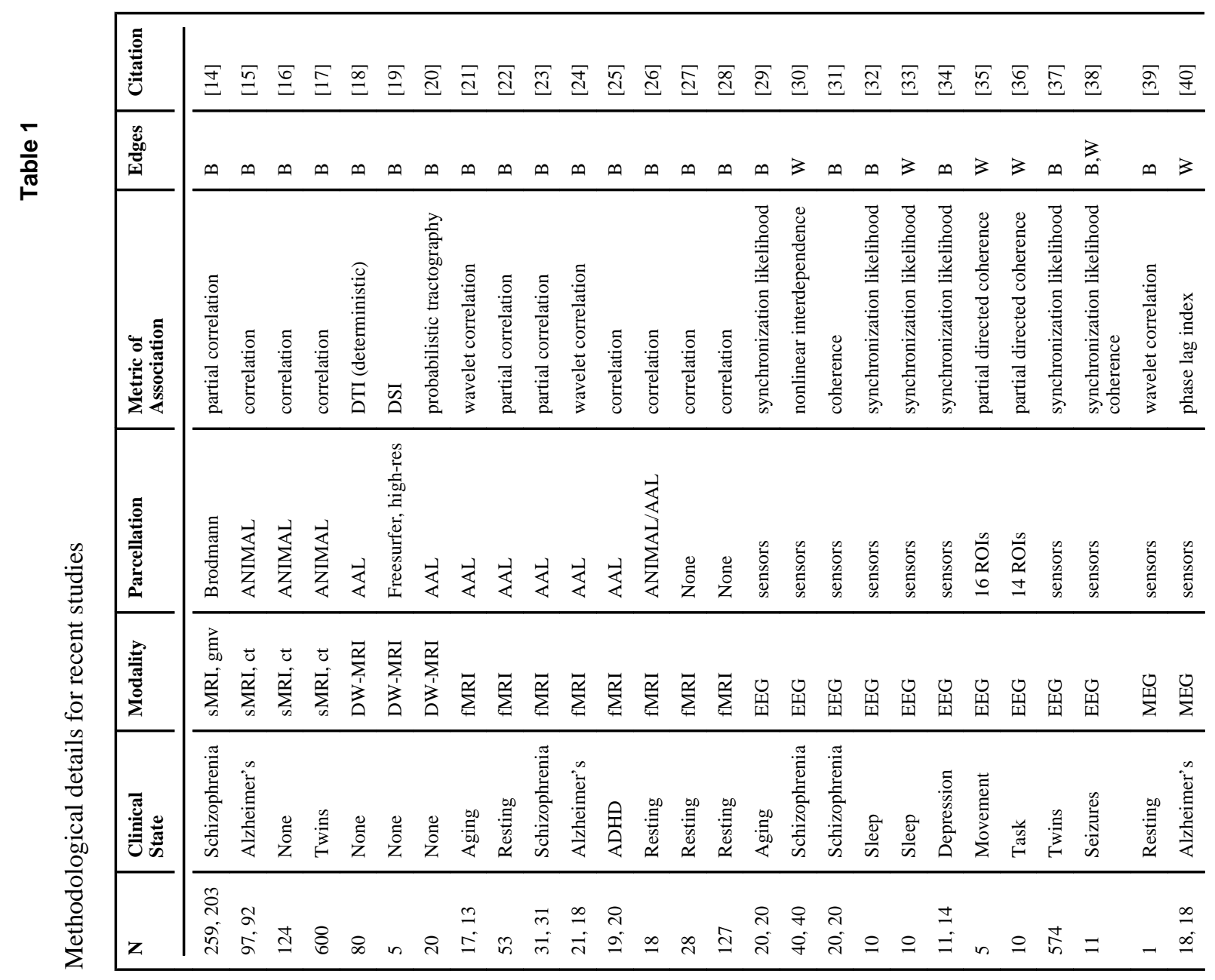

Curr Opin Neurol. Author manuscript; available in PMC 2010 August 1. 


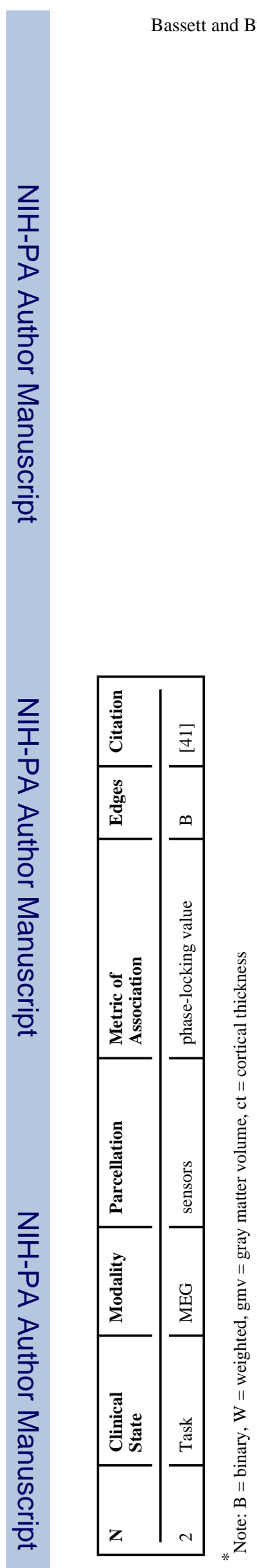

Page 14 